\title{
Prevalence of inhalant allergic rhinitis in children with adenoid hypertrophy: a prospective radioallergosorbent test (rast) study
}

\begin{abstract}
Introduction: Children with allergic rhinitis are susceptible to adenoidal hypertrophy when compared to children with no allergies. We conducted this study to determine the prevalence of inhalant allergic rhinitis among children aged 2 to 10years admitted for adenoidectomy by performing radioallergosorbent test (RAST).
\end{abstract}

Material and Methods: 75 children who underwent adenoidectomy at Hamad Medical Corporation, Doha, Qatar were recruited prospectively. 41 (54.7\%) were males and 34 $(45.3 \%)$ were females with a mean age of $4.9 \pm 4.5$ years. Blood samples were collected for total IgE and allergen specific IgE antibodies during preoperative assessment or during intravenous induction for general anesthesia.

Results: 34 (45.3\%) out of 75 children had increased total IgE, 19 (25.3\%) had positive allergen specific IgE antibodies and $7(9.3 \%)$ had both positive. Snoring $73(97.3 \%)$ was the most common symptom, followed by mouth breathing $72(96 \%)$, nasal blockage 61 $(81.5 \%)$, rhinorrhea $34(45.3 \%)$ and sneezing 11 (14.7\%). Association of nasal symptoms with total $\mathrm{IgE}$ and allergen specific $\mathrm{IgE}$ antibodies showed no statistical significance. Association of asthma 29 (38.7\%), eczema 10 (13.3\%) and family history $31(41.3 \%)$ were also not statistically significant.

Conclusion: The result of our study suggests high prevalence of allergy sensitization among children who underwent adenoidectomy but poorly correlated with nasal symptoms. However, snoring and mouth breathing were found to be statistically significant $(\mathrm{p}=0.01)$ and specific for adenoid hypertrophy in patients without allergic rhinitis. In addition, nasal blockage is more specific $(\mathrm{p}=0.02)$ for with normal total serum $\mathrm{IgE}$ levels.

Keywords: adenoidectomy, allergic rhinitis, rast, prevalence, pediatric, immunoglobulin
Volume 4 Issue 5 - 2016

\author{
Shanmugam Ganesan,' Hamad Al-Saey,' \\ Ameena Al-Bouainain, ${ }^{2}$ Aisha Larem, ${ }^{\prime}$ \\ Mohammed Hussein Magdi, ${ }^{3}$ Rajvir Singh, ${ }^{4}$ \\ Hassan Haidar' \\ 'Department of Surgery, Hamad Medical Corporation (HMC), \\ Qatar \\ 2Department of Laboratory Medicine and Pathology, HMC, \\ Qatar \\ ${ }^{3}$ Anesthesia Department, Hamad Medical Corporation (HMC), \\ Qatar \\ ${ }^{4}$ Cardiology Research Unit, Heart Hospital, Hamad Medical \\ Corporation (HMC), Qatar
}

\begin{abstract}
Correspondence: Shanmugam Ganesan, Senior Consultant, ORL-HNS (ENT) Section, Department of Surgery, Hamad Medical Corporation (HMC), Doha, Qatar, Tel +974-5588470I,
\end{abstract} Email sganesan@hamad.qa

Received: June 22, 2016 | Published: September 19, 2016
Abbreviations: SPT, skin prick test; RAST, radioallergosorbent test; AR, allergic rhinitis; AH, adenoidal hypertrophy; SPSS, statistical packages for social sciences

\section{Introduction}

Allergic diseases are one of the most frequent disorders in the pediatric population all over the world. It is known that children with allergic rhinitis (AR) are more susceptible to adenoidal hypertrophy (AH) than non-allergic children. ${ }^{1-5} \mathrm{AR}$ and $\mathrm{AH}$ in children are one of the most frequent disorders that Otolaryngologists encounter in their practice; both conditions have very similar clinical manifestations, mainly as nasal obstruction and snoring and are associated with impaired quality of life, failure to thrive and poor school performance. ${ }^{2,3,6,9}$

Strong predictors of development of AR are genetic and environmental factors with a positive family history.

Diagnosis of AR is made based on history, clinical examination and by performing allergic tests for inhalant allergens. ${ }^{6-8} \mathrm{AH}$ is a common condition in children and adenoid removal is necessary in symptomatic patients. AR and AH can co-exist in the same patient and treating allergy may relieve the patients symptoms and thus avoid unnecessary surgery. ${ }^{10,11}$ Despite this strong correlation, allergy tests are still not routinely performed in patients before adenoid surgery.

Up to our knowledge, no study has been conducted to determine the prevalence of inhalant AR in children who underwent adenoi- dectomy. The purpose of this study is to determine the prevalence of inhalant AR among children who underwent adenoidectomy by performing radioallergosorbent test (RAST).

\section{Materials and methods}

We conducted a prospective study in our department from June 2007 to December 2010. Our proposal was approved by the Ethics and Research Committee of Hamad Medical Corporation (Ref RC/1055). Children between the ages of 2 to 10 , admitted for adenoidectomy as a day care procedure were recruited for the study. Proper consents and assents were taken for involvement in the study and data was kept confidential in accordance with IRB standards.

The following data was collected preoperatively from the parents of all children regarding age, gender, nationality, nasal symptoms, asthma, eczema, family history, previous ENT surgeries and general medical problems. Children with obstructive sleep apnea, hematological disorders, immunodeficiency diseases, craniofacial syndromes, history of previous adenoidectomy and those who needed tonsillectomy were excluded from the study. Blood samples were collected, from included children, for serum total $\mathrm{IgE}$ and serum allergen specific $\operatorname{IgE}$ antibodies to inhalant allergens at the time of preoperative assessment or during intravenous induction for general anesthesia.

Data was coded and entered into a computer and processed using the Statistical Packages for Social Sciences SPSS, (windows version 21.0). Descriptive statistics in the form of mean, SD, range for in- 
terval variables, frequency and percentages for categorical variables have been performed to see an association between allergy positive and negative according to positive serum allergen specific IgE antibodies and elevated serum total IgE levels with asthma, eczema, family history of allergy and nasal symptoms, chi-square tests were performed. $\mathrm{P}$ value $\leq 0.05$ (two tailed) was considered as statistically significant level.

\section{Results}

75 children were included in our study; (Table 1) shows the demographic and clinical characteristics of allergy among included children. $41(54.7 \%)$ patients were males and $34(45.3 \%)$ were females with a mean age of $4.9 \pm 4.5$ years. 19 out of $75(25.3 \%$; $95 \%$ Confidence Interval of $16 \%$ to $37 \%$ ) had increased serum allergen specific IgE antibodies (positive RAST) to inhalant allergens and 34 (45.3\%) out of 75 children had elevated serum total IgE levels. Both serum allergen specific IgE antibodies and serum total IgE results were positive in $7(9.3 \%)$ patients. Snoring $(97.3 \%)$ was the most common symptom, followed by mouth breathing (96\%), nasal blockage (81.5\%), rhinorrhea (45.3\%) and sneezing (14.7\%). Association of nasal symp- toms with serum total $\operatorname{IgE}$ and serum allergen specific $\operatorname{IgE}$ antibodies showed no statistical significance between the two (Table 2). Association of asthma (38.7\%), eczema (13.3\%) and family history (41.3\%) were also not found to be statistically significant (Table 3). Higher proportion of snoring and mouth breathing were found in negative serum allergen specific $\operatorname{IgE}$ antibody group $(\mathrm{p}=0.01)$ and a higher percentage of nasal blockage was found in patients with normal serum total $\operatorname{IgE}(\mathrm{p}=0.02)$ (Tables $2 \& 3)$.

Table I Demographic and clinical characteristics of allergy among children admitted for adenoidectomy

\begin{tabular}{ll}
\hline Variables & $\mathbf{N}(\%)$ \\
\hline Age & $4.9 \pm 4.5$, Range (2-I0 Years) \\
\hline Gender & Male: 4I(54.7\%); \\
\hline & Female: $34(45.3 \%)$ \\
\hline Asthma & $29(38.7 \%)$ \\
\hline Eczema & I0(I3.3\%) \\
\hline Family History & $31(4 I .3 \%)$ \\
\hline Total IgE & $34(45.3 \%)$ \\
\hline Serum Specific IgE & $19(25.3 \%)$ \\
\hline
\end{tabular}

Table 2 Nasal symptoms and their association with serum total lgE and serum allergen specific lgE antibodies

\begin{tabular}{|c|c|c|c|c|c|c|c|}
\hline \multirow[t]{2}{*}{ Variable } & \multirow[b]{2}{*}{ Total (\%) } & \multicolumn{3}{|c|}{ Serum Allergen Specific IgE Antibodies } & \multicolumn{3}{|c|}{ Serum Total IgE } \\
\hline & & Positive (\%) & Negative (\%) & $P$ value $(\%)$ & Abnormal (\%) & Normal (\%) & $P$ value \\
\hline Snoring & $73(97.3)$ & $19(26.1)$ & $54(73.9)$ & 0.01 & $3 I(96.9)$ & $42(97.7)$ & 83 \\
\hline Nasal Blockage & $6 I(8 I .5)$ & $16(84.2)$ & $45(80.4)$ & 0.71 & $22(68.8)$ & $39(90.7)$ & 0.02 \\
\hline Rhinorrhea & $34(45.3)$ & $9(47.4)$ & $25(44.6)$ & 0.84 & | 4(43.8) & $20(26.5)$ & 0.81 \\
\hline Mouth Breathing & $72(96.0)$ & $19(26.4)$ & $53(73.6)$ & 0.01 & $30(93.8)$ & 42(97.7) & 0.4 \\
\hline Sneezing & $\mathrm{II}(\mathrm{I} 4.7)$ & $2(10.5)$ & $9(16.1)$ & 0.56 & $5(15.6)$ & $6(14.0)$ & 0.84 \\
\hline
\end{tabular}

Table 3 Clinical characteristics and their association with serum total IgE and serum allergen specific IgE antibodies

\begin{tabular}{|c|c|c|c|c|c|c|c|}
\hline \multirow[t]{2}{*}{ Variable } & \multirow[b]{2}{*}{ Total } & \multicolumn{3}{|c|}{ Serum Allergen Specific IgE Antibodies } & \multicolumn{3}{|c|}{ Serum Total IgE } \\
\hline & & Positive (\%) & Negative (\%) & PValue & Abnormal (\%) & Normal (\%) & PValue \\
\hline Asthma & 29 & $7(36.7)$ & $22(39.3)$ & 0.85 & $13(40.6)$ & $16(37.2)$ & 0.76 \\
\hline Eczema & 10 & $4(21.1)$ & $6(10.7)$ & 0.25 & $5(15.6)$ & $5(11.6)$ & 0.62 \\
\hline Family History & 31 & $10(52.6)$ & $21(37.5)$ & 0.25 & $15(46.9)$ & 16(37.2) & 0.4 \\
\hline
\end{tabular}

\section{Discussion}

The prevalence of IgE mediated AR has increased dramatically over the past few decades due to environmental, urbanization and socio-economic factors. Sensitivity to food and inhalant allergens can occur as early as the first 6 to 12 months of life. Approximately $40 \%$ of children have AR. ${ }^{6}$ Exposure to inhalant allergic protein could prime the immune systems of genetically predisposed infants. High risk children are those with history of sensitization to egg or milk proteins, asthma and eczema as well as parents with AR, asthma and eczema. Environmental co-factors that increase the risk of AR are: exposure to pets reduced indoor ventilation, outdoor air pollution, exposure to passive smoking and dietary factors.

$\mathrm{AH}$ is one of the most common childhood otolaryngology disorders clinically present with snoring, mouth breathing, nasal blockage and rhinorrhea. Adenoidal size increases with age up to 5 to 6years and then decreases gradually by the age of 8 to 9years. ${ }^{1}$ Many of the symptoms of AH and AR are similar and it is likely that only one condition is often recognized, which can lead to misdiagnosis and consequently wrong treatment. The causes of $\mathrm{AH}$ are not fully known but literature evidence suggests an association between $\mathrm{AR}$ and $\mathrm{AH} .{ }^{1-5}$ Modrzynski et al., ${ }^{3}$ showed that $\mathrm{AH}$ was more prevalent in children with AR and appear to have greater susceptibility to AH than non-allergic children. ${ }^{2}$

Our study among children aged 2 to 10 years who were admitted for adenoidectomy operation, showed high prevalence $(25.3 \%)$ of sensitization to inhalant allergens. Similar observations noted in a questionnaire based cross sectional population study in 2006, had shown prevalence of diagnosed AR, asthma and eczema among school children aged 6 to 14years were 30.5, 19.8 and 22.5\%. ${ }^{12}$ Another study by Kerkadi et al., ${ }^{13}$ in 2009 revealed high prevalence of sensitization to inhalant allergens (19.4\%) and food allergens (29.9\%) among children aged 1month to 10years who attended the Allergy and Asthma Paediatric Clinic in Qatar. ${ }^{13}$ The consanguinity rate among the local population is very high $(54 \%)$ and hence, diseases with genetic influences are also expected to be high in this group of population. ${ }^{12}$ Life style changes, urbanization, rapid industrialization and increase in domestication of pets may have contributed to the high prevalence rate of sensitization to inhalant allergens among children.

Various in-vitro and in-vivo tests have been described in the literature for diagnosis of AR in children. ${ }^{6-8}$ There is currently no single gold standard test for diagnosing inhalant allergies. ${ }^{7}$ Clinical history makes a vital link between allergy tests and allergic diseases. The 
most commonly used methods for diagnosing allergen sensitization are measurement of serum allergen specific IgE antibodies and skin prick test (SPT). Two of these methods have similar diagnostic value in terms of sensitivity and specificity, which vary with the clinical scenario and allergen tested. ${ }^{6}$ Some studies have found SPT to have a lower false negative rate (more sensitive) than serum allergen specific IgE antibody assay. ${ }^{10}$ Similarly, serum allergen specific IgE antibodies were found to have fewer false positive rates (more specific) compared to SPT. ${ }^{6,7}$ In our study, we performed serum allergen specific IgE antibody assay using either Phadia ImmunoCAP or RIDA Allergy screen test panel for in vitro diagnostics (R-Biopharm) which included specific allergens in the environment. We selected in vitro assay for our patients as it is most suitable for young children and in avoiding phobia of needles as blood samples for the test are collected at the time of anesthesia induction or during time of preoperative assessment for surgery. There is also no risk of anaphylaxis or dermographism, not influenced by recent anti-allergic medications and it abolishes interpreter errors. A negative test virtually rules out AR in the study group.

Serum total IgE level is helpful in diagnosis of allergic diseases but poorly correlated with clinical disease and is non-specific. Our study showed 34 out of 75 children (45.3\%) had increased IgE level which signifies allergies in the group of patients tested but is poorly correlated with inhalant allergens. Both serum allergen specific IgE antibody assay and IgE levels were positive in $7(9.3 \%)$ patients. Association of nasal symptoms with the total $\mathrm{IgE}$ and serum allergen specific $\operatorname{IgE}$ antibody assay was not statistically significant except snoring and mouth breathing were found to be more statistically significant $(p=0.01)$ in negative serum allergen specific IgE assay group. This signifies that these two symptoms are more specific for $\mathrm{AH}$ in patients without AR. A similar finding was noted in the nasal blockage group where nasal blockage was associated with normal total $\operatorname{IgE}$ levels $(\mathrm{p}=0.02)$ which signifies that nasal blockage is more prevalent among AH patients than patients with AR. Association of asthma (38.7\%), eczema $(13.3 \%)$ and family history of AR, asthma and eczema (41.3\%) were found not statistically significant in the study. The limitation of the study was that quantitative analysis of individual allergens was not analyzed. However, all positive allergy patients with significant allergy history were treated with anti-allergic measures during post-operative follow up.

\section{Conclusion}

Our study showed high prevalence of sensitization to inhalant allergens among children admitted for adenoidectomy surgery without association for nasal symptoms, asthma, eczema and family history. The result of our study suggests high prevalence of allergy sensitization among children who underwent adenoidectomy but poorly correlated with nasal symptoms. However, snoring and mouth breathing were found to be statistically significant and specific for $\mathrm{AH}$ in patients without AR. In addition, nasal blockage is more specific for AH with normal total serum IgE levels. We suggest a larger sample size with adequate control subjects which would help provide more evidence in support of allergy being a major contributor in $\mathrm{AH}$ patients who require adenoidectomy.

\section{Oral presentation done at the ERS meeting}

The paper was presented as a short oral presentation at the European Rhinological Society meeting (ERS 2014) in Amsterdam, the Netherlands, June 22-26, 2014.

\section{Acknowledgements}

We would like to express our sincere gratitude towards the Medical Research Centre, Hamad Medical Corporation, Doha, Qatar for the financial support provided for the study and for the secretarial support provided for the data analysis.

\section{Conflicts of interest}

Author declares there are no conflicts of interest.

\section{Funding}

None.

\section{References}

1. Scadding G. Non-surgical treatment of adenoidal hypertrophy: the role of treating IgE-mediated inflammation. Pediatr Allergy Immunol. 2010;21(8):1095-1096.

2. Ibáñez MD, Valero AL, Montoro J, et al. Analysis of comorbidities and therapeutic approach for allergic rhinitis in a pediatric population in Spain. Pediatr Allergy Immunol. 2013;24(7):678-684.

3. Modrzynski M, Zawisza E. The influence of birch pollination on the adenoid size in children with intermittent allergic rhinitis. Int J Pediatr Otorhinolaryngol . 2007;71(7):1017-1023.

4. Olusesi AD, Undie NB, Amodu JE. Allergy history as a predictor of early onset adenoids/adenotonsillar hypertrophy among Nigerian children. Int J Pediatr Otorhinolaryngol. 2013;77(6):1032-1035.

5. Marseglia GL, Poddighe D, Caimmi D, et al. Role of adenoids and adenoiditis in children with allergy and otitis media. Curr Allergy Asthma Rep. 2009;9(6):460-464

6. Cox L. () Overview of serological-specific $\operatorname{IgE}$ antibody testing in children. Curr Allergy Asthma Rep. 2011;11(6):447-453.

7. Cox L, Williams B, Sicherer S, et al. Pearls and pitfalls of allergy diagnostic testing: report from the American College of Allergy, Asthma and Immunology/American Academy of Allergy, Asthma and Immunology Specific IgE Test Task Force. Ann Allergy Asthma Immunol . 2008;101(6):580-592.

8. Schäfer T, Hoelscher B, Adam H, et al. Hay fever and predictive value of prick test and specific IgE antibodies: a prospective study in children. Pediatr Allergy Immunol. 2003;14(2):120-129.

9. Sih T, Mion O. Allergic rhinitis in the child and associated comorbidities. Pediatr Allergy Immunol. 2010;21(1 Pt 2):e107-e113.

10. Huang SW, Giannoni C. The risk of adenoid hypertrophy in children with allergic rhinitis. Ann Allergy Asthma Immunol. 2001;87(4):350-355.

11. Lee DH. Is there any correlation between allergy and adenotonsillar tissue hypertrophy? Int J Pediatr Otorhinolaryngol. 2012;76(2):303.

12. Janahi IA, Bener A, Bush A. Prevalence of asthma among Qatari schoolchildren: International Study of Asthma and Allergies in Childhood, Qatar. Pediatr Pulmonol. 2006;41(1):80-86.

13. Kerkadi A, Althani A, Al-Boainain, et al. Sensitization to common food and inhalant allergens in children attending Allergy and Asthma Pediatric Clinic in Doha, Qatar. Journal of Food, Agriculture \& Environment. 2009; 7(2):213-217. 